\title{
Comparison of the Effectiveness of Music Video Therapy and Music Therapy on Pain after Cardiothoracic Surgery in Preschool Children
}

\author{
Ya-Li Huang, MM, ${ }^{1,2,3,4}$ Yu-Qing Lei, MM, ${ }^{1,2,3,4}$ Jian-Feng Liu, MM, ${ }^{1,2,3,4}$ Hua Cao, MD, ${ }^{1,2,3,4}$ \\ Xian-Rong, Yu, MM, 1,2,3,4* Qiang Chen, MD ${ }^{1,2,3,4 *}$ \\ ${ }^{1}$ Department of Cardiac Surgery, Fujian Maternity and Child Health Hospital, Affiliated Hospital of Fujian Medical University, \\ Fuzhou, China; ${ }^{2}$ Fujian Key Laboratory of Women and Children's Critical Diseases Research, Fujian Maternity and Child Health \\ Hospital, Fuzhou, China; ${ }^{3}$ Fujian Branch of Shanghai Children's Medical Center Affiliated to Shanghai Jiaotong University School of \\ Medicine, Fuzhou, China, ${ }^{4}$ Fujian Children's Hospital, Fuzhou, China
}

\section{ABSTRACT}

Objective: To explore the effects of music video therapy on pain among preschool children after cardiothoracic surgery.

Methods: Patients in the music video therapy (MVT) group received a 30 -min music video intervention, while patients in the music therapy (MT) group received a 30-min musical intervention. Both groups were given their respective therapy three times a day for three days. Patients in the control group did not receive MVT or MV. Measures, including pain scores, vital signs (heart rate, mean arterial pressure, respiratory rate, and oxygen saturation), and other postoperative indicators were recorded and analyzed.

Results: The MVT group showed a statistically significant decrease in heart rate, mean arterial pressure, and respiratory rate at the first day after surgery and pain scores at the first and second day after surgery compared to the MT group, but no significant difference was identified in oxygen saturation. The postoperative indicators including cumulative capacity of sufentanil use, the length of intensive care unit (ICU) stay, and the length of hospital stay in the MVT group were significantly lower than those in the control group.

Conclusion: The findings provide further evidence to support the practice of music video therapy as a non-pharmaceutical intervention to reduce postoperative pain, reduce the dosage of analgesics, shorten the length of ICU and hospital stay in preschool children after the cardiothoracic surgery.

\section{INTRODUCTION}

Thoracotomy is a type of surgery said to be one of the most painful surgeries during the postoperative period [Baidya 2014]. Pain associated with cardiothoracic surgery may

Received December 2, 2020; accepted fanuary 3, 2021.

*These authors share the corresponding authorship.

Correspondence: Qiang Chen, MD, Department of Cardiac Surgery, Fujian Maternity and Child Health Hospital, Affiliated Hospital of Fujian Medical University,Fuzbou,China; (e-mail: chenqiang2228@163.com) accelerate heart rate, increase blood pressure, decrease diaphragmatic movement, and other respiratory muscles function, leading to respiratory disorders and cardiologic complications [Ziehm 2017; Bayman 2017]. This study suggested that pain affected the recovery of cardiopulmonary function after the cardiothoracic surgery, which could also seriously affect a child's quality of life, and have a series of short-term and long-term impacts [Scohy 2011; Kortesluoma 2008]. Recently, there is more research about postoperative pain in children, calling for clinic medical professionals' attention [Zieliński 2020]. The treatment of postoperative analgesia tends to reduce the dosage of opioids, and even some scholars have put forward the goal of "opioid-free analgesia" [Yap 2020]. Music therapy, as a non-pharmaceutical adjuvant, has been used to alleviate pain after cardiac surgery [Hole 2015]. But music therapy may not be enough to distract children's attention from postoperative pain, especially for patients in an intensive care unit (ICU) environment. Studies have also confirmed that the effect of the audiovisual intervention was better than that of MT [Chow 2016; Custódio 2020; Oliveira 2017]. Based on children's psychological status, cognitive behavior, and development process, this study was conducted to investigate the effectiveness of MVT and to provide further evidence to support the practice of MVT for non-pharmaceutical intervention on children's postoperative pain.

\section{METHODS}

\section{Sample Size and Randomization}

$\mathrm{G}^{*}$ Power (version 3.1.9.2, Heinrich-Heine University of Dusseldorf, Germany) was used to determine the sample size [Faul 2007]. Alpha value was set at 0.05 , and a power of 0.9 , the resulting minimum sample size was 105 patients. Considering an attrition rate of $20 \%$, the total sample size required was 126 subjects. Children were randomly assigned to three groups using a computer program: the MVT group consisted of the children who received music video intervention ( $\mathrm{n}=42$ ); the MT group consisted of the children who received music therapy $(n=42)$; the control group consisted of the children who did not receive MT or MVT $(n=42)$. There was no blinding for the researcher. 
Table 1. Demographic and Baseline Characteristics of Participants

\begin{tabular}{|c|c|c|c|c|}
\hline Age, $y$ & $5.1 \pm 1.81$ & $4.8 \pm 1.74$ & $5.0 \pm 1.75$ & .802 \\
\hline Weight, kg) & $16.0 \pm 3.61$ & $17.5 \pm 3.74$ & $16.9 \pm 4.09$ & .689 \\
\hline Male sex, n (\%) & $26(61.9)$ & $25(59.5)$ & $27(64.3)$ & .904 \\
\hline Female sex, $\mathrm{n}(\%)$ & $16(38.1)$ & $17(40.5)$ & $15(35.7)$ & \\
\hline Rural area & $24(57.1)$ & $28(66.7)$ & $23(54.8)$ & .501 \\
\hline City & $18(42.9)$ & $14(33.3)$ & $19(45.2)$ & \\
\hline \multicolumn{5}{|l|}{ Only child, n (\%) } \\
\hline Yes & $26(61.9)$ & $23(54.8)$ & $22(52.4)$ & .657 \\
\hline Left side & $6(14.3)$ & $10(23.8)$ & $11(26.2)$ & \\
\hline Right side & $9(21.4)$ & $8(19.1)$ & $9(21.4)$ & \\
\hline Operation time, hours & $3.46 \pm 0.26$ & $3.35 \pm 0.33$ & $3.40 \pm 0.28$ & .748 \\
\hline Anesthesia time, hours & $4.24 \pm 0.40$ & $4.19 \pm 0.38$ & $4.22 \pm 0.43$ & .802 \\
\hline Duration of mechanical ventilation, hours & $3.58 \pm 0.81$ & $3.64 \pm 0.73$ & $3.85 \pm 0.92$ & .214 \\
\hline
\end{tabular}

\section{Participant Recruitment}

Two tertiary hospitals provided the setting from June 2019 to July 2020 for this study. Inclusion criteria for participants included: (a) underwent thoracotomy; (b) aged from 3 to 7 years; (c) able to understand, see, and listen; (d) hemodynamics stable after operation; (e) tracheal extubation; (f) completed the informed consent from parents. Exclusion criteria were as follows: (a) primary disease complicated with other diseases; (b) history of chronic pain; (c) blindness and hearing deficits; (d) postoperative complication (atelectasis, pneumonia, heart failure, etc.); (e) previous surgical history; (f) inability to complete the study. The research ethics committee approved the study at our hospital.

\section{Measurements}

Vital signs were measured at each test point, including heart rate (HR), mean arterial pressure (MAP), respiratory rate (RR), and arterial oxygen saturation $\left(\mathrm{SpO}_{2}\right)$. The pain was measured using Wong-Baker FACES pain rating scale and Face, Legs, Activity, Cry, Consolability (FLACC) behavioral pain assessment scale. WB-FACES pain rating scale was a kind of self-assessment method suitable for children over three years old [Ozdemir 2020]. This scale did not require reading, writing, and expression, which was also suitable for children with acute pain [Garra 2010]. There were six faces on this scale, representing pain intensity from 0 to 10 . The left-most face was marked as 0 and showed no pain, whereas the face on the far right marked as 10 showed the most severe pain with the numbers going up in twos $(0,2,4,6,8,10)$. As the score increased, pain tolerance decreased. Before the procedure, the participant children were informed about the way to use this scale. The children were told to choose the face that best expressed their feelings. The FLACC behavioral pain assessment scale was composed of five items and suitable for children aged 0-18 years [Von Baeyer 2007]. Each item represented different pain behavior, and five items were scored from 0 to 10 points. The clinical staff observed for 2 minutes to evaluate the pain level of the children. The result showed that FLACC scores $\leq 3$ indicated a little bit of pain or no pain; 4-7 indicated moderate pain; 8-10 indicated severe pain. It was often used to measure postoperative pain in children [Voepel-Lewis 2002; Malviya 2006]. The FLACC scale could express good evidence of test-retest and inter-rater reliability and constructed validity among the children.

\section{Analgesia Protocol and Intervention}

After surgery, the children were transferred to the cardiothoracic ICU, and standard monitoring including electrocardiography, pulse oximetry, and blood pressure monitoring were performed. A standard postoperative analgesia strategy was used for all children. When the patients were fully awake, the endotracheal tube was removed, and a postoperative analgesia pump (sufentanil $2.5 \mu \mathrm{g} / \mathrm{kg}$, tropisetron $0.2 \mathrm{mg} / \mathrm{kg}$, and physiological saline $100 \mathrm{ml}$ ) was used in all patients. The initial dose was $2 \mathrm{ml}$, and the regular infusion rate was $2 \mathrm{ml} / \mathrm{h}$; the pressing dose was $0.5 \mathrm{ml}$. A team of ICU doctors and nurses assessed the patients' analgesic scores and determined the analgesic pump's speed and whether to increase the number of presses. When the patient experienced severe pain (Wong-Baker facial scale 
Table 2. Comparison of Hemodynamics between the Three Groups

\begin{tabular}{|c|c|c|c|c|c|}
\hline \multirow[t]{4}{*}{$\mathrm{HR}$, beats/min } & Pre-test & $113 \pm 12.15$ & $112 \pm 11.63$ & $113 \pm 13.03$ & .842 \\
\hline & 1st Post-test & $101 \pm 10.52$ & $113 \pm 12.32$ * & $119 \pm 14.52 *$ & .016 \\
\hline & 2nd Post-test & $99 \pm 9.27$ & $101 \pm 9.64$ & $114 \pm 11.74 *$ & .034 \\
\hline & 3rd Post-test & $97 \pm 7.98$ & $98 \pm 8.37$ & $110 \pm 10.38 *$ & .046 \\
\hline \multirow{3}{*}{ MAP, mmHg } & 1st Post-test & $68 \pm 7.43$ & $75 \pm 7.74$ * & $76 \pm 8.31 *$ & .025 \\
\hline & 2nd Post-test & $69 \pm 6.39$ & $70 \pm 6.26$ & $73 \pm 7.59 *$ & .043 \\
\hline & 3rd Post-test & $67 \pm 6.72$ & $68 \pm 6.95$ & $71 \pm 6.53^{*}$ & .047 \\
\hline $\mathrm{RR}$, rates/min & Pre-test & $28 \pm 1.82$ & $27 \pm 1.76$ & $28 \pm 1.98$ & .842 \\
\hline \multirow[t]{4}{*}{$\mathrm{SpO}_{2}, \%$} & Pre-test & $98 \pm 2.64$ & $97 \pm 2.15$ & $98 \pm 2.43$ & .786 \\
\hline & 1st Post-test & $98 \pm 3.29$ & $98 \pm 2.37$ & $98 \pm 2.99$ & .848 \\
\hline & 2nd Post-test & $98 \pm 2.11$ & $98 \pm 2.02$ & $98 \pm 2.63$ & .853 \\
\hline & 3rd Post-test & $98 \pm 2.02$ & $98 \pm 2.14$ & $98 \pm 2.40$ & .867 \\
\hline
\end{tabular}

*Indicates $P<.05$ compared with the MVT group.

score $\geq 6$ or FLACC score $\geq 4$ ), increasing the dose or applying press was initiated. No additional analgesics were used in any patients. At the same time, MVT or MT was administered according to the different groups.

The researcher prepared the environment sufficiently to eliminate distractions and tried to keep the children from being disturbed. Three to five favorite children's MV were chosen and transferred to smartphones before the intervention. In the MVT group, MV was provided for 30 minutes at times when usually less clinical interventions were scheduled; in the morning (07:30-08:00 hr), at noon (12:00-12:30 hr), and evening (21:30-22:00 hr), three times a day for three days. The sound was controlled at 30-50 dB. The sight distance was kept 40-60 $\mathrm{cm}$ as far as possible and adjusted to the comfortable posture, with headphones connected. Simultaneously, the children in the MT group using the same smartphones only listened to their favorite music for 30 minutes each time, three times a day. In the control group, no MVT or MT was used in the patients.

\section{Data Collection}

The general data included age, sex, weight, residence, only child or not, the operation time, the anesthesia time, and mechanical ventilation duration. The pre-test (before intervention), pain scores, and vital signs were collected. The first day after surgery (1st Post-test), the second day after surgery (2nd Post-test) and the third day after surgery (3rd Post-test), the pain scores, vital signs, the cumulative capacity of sufentanil use, the length of ICU and hospital stay, and hospitalization cost were recorded.

\section{Statistical Analysis}

Data were analyzed using SPSS (version 21.0 for Windows, IBM Corporation, Armonk, NY, USA). Participant characteristics and the survey results were analyzed with descriptive statistics and were determined using categorical variables' frequency distributions and mean plus/minus standard deviation for continuous variables. Measurements of the three time points of the same day after the intervention were summed, and the average scores of each child were calculated. ANOVA was used to compare the mean of the three groups, and the S-N$\mathrm{K}(\mathrm{S})$ method was used to compare each pair. The Pearson chisquare or Fisher test was used to categorize the variables. A $P$ value of $<.05$ was defined as statistically significant.

\section{RESULTS}

One hundred and twenty-six children participated in the study. Table 1 presents the demographic and baseline characteristics of these children. No statistically significant difference was found among the three groups $(P>.05)$. Table 2 listed the three groups' characteristics in HR, MAP, RR, and $\mathrm{SpO}_{2}$ of pre-test and post-test data. Before the intervention, there were no statistical differences in vital signs. After the intervention, there were statistical differences of HR, MAP, and RR among the three groups, but no significant difference existed with $\mathrm{SpO}_{2}$. On the first day after surgery, HR, MAP, and RR in the MVT group were lower than those in the MT group $(P<.05)$. 
Table 3. Comparison of Wong-Baker FACES Scores of the Three Groups

\begin{tabular}{lcccc}
\hline & MVT Group & MT Group & Control Group & $P$ \\
\hline Pre-test & $5.9 \pm 1.91$ & $6.0 \pm 1.86$ & $6.0 \pm 1.86$ & .941 \\
1st Post-test & $5.1 \pm 1.72 *$ & $6.1 \pm 1.92 \dagger$ & $6.2 \pm 2.04 \dagger$ & .034 \\
2nd Post-test & $2.4 \pm 0.98 *$ & $3.7 \pm 1.56^{*} \dagger$ & $5.4 \pm 1.63 \dagger$ & .023 \\
3rd Post-test & $1.2 \pm 1.48 *$ & $1.5 \pm 1.77^{*}$ & $3.4 \pm 1.76^{*} \dagger$ & .019 \\
\hline
\end{tabular}

*Indicates $P<.05$ compared with Pre-test; findicates $P<.05$ compared with the MVT group.

The evaluation of the Wong-Baker FACES and FLACC scores of the children are presented in Table 3 and Table 4. There was a statistical difference among the three groups after the intervention $(P<.05)$, and there was a statistically significant difference in the MVT and MT groups on the first day and second day after surgery $(P<.05)$. Results also revealed that the longer it had been after the surgery, the fewer children reported their pain, but the decline of WongBaker FACES and FLACC scale scores at three-time points after intervention in the MVT group were better than those in the MT group.

The postoperative indicators including the cumulative capacity of sufentanil use, the length of ICU stay, and the length of hospital stay in the control group were significantly higher than those in the MVT group and the MT group $(P=$ $.046, P=.042$, and $P=.037$, respectively). Although the above three indicators in the MVT group were better than those in the MT group, the difference was not statistically significant. There was no statistically significant difference in hospitalization cost among the three groups (Table 5).

\section{DISCUSSION}

Pain after cardiothoracic surgery can be debilitating to patients and lead to poor outcomes, including respiratory complications such as atelectasis and pneumonia, more extended hospital stays, low quality of life, and chronic persistent postoperative pain syndrome [Marshall 2020]. The most frequent pain sources after these procedures include surgical incision, rib damage or resection, chest tubes, and the suturing technique. Pain after cardiothoracic surgery usually lasts approximately 72-96 hours and is more severe than pain after other body areas' surgeries [Wojtyś 2019]. Management of postoperative pain has become a significant concern in cardiothoracic surgery, especially for those pediatric patients. Ineffective pain management results in hemodynamic perturbations with systemic complications, including pulmonary (atelectasis, pneumonia, and stasis of bronchial secretions), cardiovascular (increased oxygen consumption and tachycardia), musculoskeletal (muscle weakness), and increased neurohormonal response [Bayman 2017]. Effective
Table 4. Comparison of FLACC Scores of the Three Groups

\begin{tabular}{lcccc}
\hline & MVT Group & MT Group & Control Group & $P$ \\
\hline Pre-test & $5.8 \pm 1.85$ & $5.9 \pm 1.82$ & $6.0 \pm 1.84$ & .897 \\
1st Post-test & $5.1 \pm 1.76 *$ & $6.0 \pm 1.87 \dagger$ & $6.1 \pm 1.79 \dagger$ & .042 \\
2nd Post-test & $2.5 \pm 1.24 *$ & $3.7 \pm 1.82 * \dagger$ & $4.2 \pm 1.90 * \dagger$ & .033 \\
3rd Post-test & $1.3 \pm 1.51 *$ & $1.5 \pm 1.63^{*}$ & $3.2 \pm 1.68 * \dagger$ & .016 \\
\hline
\end{tabular}

*Indicates $P<.05$ compared with Pre-test; †indicates $P<.05$ compared with the MVT group.

treatment of post-thoracotomy pain is particularly important to keep children comfortable, alleviate pain, shorten hospital stay, and minimize complications [Savva 2019]. Selection of a multimodal approach to postoperative pain management is advocated, including non-pharmaceutical methods, reducing the use of analgesics, improving acute pain, and reducing conversion to chronic pain [Bigeleisen 2015].

Music therapy is a non-pharmaceutical method to alleviate pain, which is free of side effects. The most extensive systematic review of music and postoperative recovery showed that music could improve outcomes and was significantly associated with reduced pain, anxiety, analgesia usage, and increased patient satisfaction [Lin 2020; Hole 2015]. The brain structure and internal inhibition function of preschool children are developing rapidly in the stage of thinking in images [Houdé 2011; Hammond 2014]. This stage is characterized by the dominance of unintentional attention and the gradual development of intentional attention. Children's attention can be attracted to objects with strong intuition, with incredibly significant perceptual changes. Vision is an essential sensation for individuals, which provides much information, followed by a sense of hearing and touch. With the popularization and development of Internet technology, smartphones, and other media, these tools have become essential carriers in children's lives. Combining the psychological needs of preschool children, the music video is employed based on music therapy and applied to relieve the pain of preschool children after cardiothoracic surgery. Studies found that watching a video was more effective than other active distraction in easing childhood patients' pain [Weydert 2006; Bellieni 2006; Salmon 2006]. The results of this study also showed that music videos were more likely to attract children's attention and acted better than music therapy in relieving postoperative pain after cardiothoracic surgery.

In this study, vital signs, and subjective and objective behavior assessment of pain were used to evaluate the degree of pain and intervention effect. Vital signs were the response to postoperative pain, but drug effects or other stress reactions also had similar changes, so it had no specificity and was for reference only. Table 2 shows that hemodynamic indexes of the three groups gradually tended to be stable as time went on after surgery, but HR, MAP, and RR in the MVT group were more stable than those in the MT group on the first day after 
Table 5. Comparison of Postoperative Conditions among the Three Groups

\begin{tabular}{lcccc}
\hline & MVT Group & MT Group & Control Group \\
\hline Cumulative capacity of sufentanil use, $\mathrm{ml}$ & $44.87 \pm 3.89$ & $46.13 \pm 4.24$ & $50.64 \pm 6.53^{*} \dagger$ & .046 \\
Length of ICU stay, d & $1.2 \pm 0.63$ & $1.4 \pm 0.81$ & $2.3 \pm 1.02 * \dagger$ & .042 \\
Length of hospital stay, d & $8.2 \pm 2.47$ & $9.3 \pm 3.55$ & $11.2 \pm 4.06^{*} \dagger$ & .037 \\
Hospitalization costs, 10,000 CNY & $5.3 \pm 1.14$ & $5.4 \pm 1.37$ & $5.6 \pm 1.73$ & .069 \\
\hline
\end{tabular}

*Indicates $P<.05$ compared with MVT group; †indicates $P<.05$ compared with MT group.

surgery, which was conducive to the recovery of cardiopulmonary function. After the mechanical ventilation was stopped, the patient was given oxygen inhalation by nasal catheter or mask, and the $\mathrm{SpO}_{2}$ of the children did not show a significant decrease before and after intervention among the three groups. Table 3 and Table 4 showed that both MT and MVT could relieve postoperative pain; the pain scores on the first day after surgery were at a high level and gradually decreased from the second day until the pain was relieved on the third day, which was related to the gradual relief of postoperative pain as time went on. The most severe pain occurred on the first day after surgery, which was consistent with other studies that showed mild to severe pain within the first three days after surgery, with the degree of pain gradually decreasing [Boric 2019]. On the first day after surgery, the pain caused by sternotomy, the change dressings of incision, and the removal of invasive arteries and catheters can cause severe pain in children.

On the second day after surgery, the pain was continuously experienced in slapping the back to stimulate expectoration and pulling out the thoracic drainage tube, but such medical care procedures were significantly less than those in the day before. The scores of Wong-Baker FACES and FLACC in the MVT group were lower than those in the MT group on the first and second day after surgery, and the decrease of pain scores in the MVT group were better than that in the MT group, which indicated that MVT intervention could relieve pain to some extent. There was no difference between these two groups on the third day, which was related to decreased medical care procedures and the gradual relief of postoperative pain. Compared with the control group, MVT and MT groups could reduce the dosage of analgesics and shorten ICU and hospital stay length. But there was also no significant difference between the MVT and MT groups, which indicated analgesics were essential as a primary treatment strategy. The non-pharmaceutical method of pain intervention could be a useful supplement, but it could not completely replace the analgesic effect. Hence, MVT intervention could effectively distract preschool children's attention from postoperative pain, relieve postoperative pain to some extent, and facilitate their postoperative recovery.

The study had the following limitations, though our study's results and others' reporting experience show that both music therapy and music video therapy affected pain relief. But we still thought of these therapies as complementary treatments, which were difficult to significantly impact hospital statistics, morbidity, mortality, and opiate consumption. The selection of evaluation indicators in this study had an absolute subjective deviation, which might have an individual impact on the results. MVT intervention is a non-pharmaceutical method, but it is ineffective when severe pain occurs. There is no unified standard for the interval time of postoperative pain assessment in children, and there is no authoritative evaluation tool to test the efficacy of music therapy. Due to the limitation of conditions, this study was not a double-blind study without multicenter and large sample, which might have a certain deviation on the data collection and arrangement, so the research results had certain limitations.

\section{Conclusion}

Music video therapy is recommended as an effective complementary therapy for preschool children after cardiothoracic surgery, which can reduce postoperative pain, reduce the dosage of analgesics, shorten the length of ICU and hospital stay, and be used as another non-pharmaceutical method of pain intervention.

\section{ACKNOWLEDGMENTS}

We gratefully acknowledge the contribution of the participating doctors: Zeng-chun Wang, Jing Wang, Yi-Rong Zheng, Ling-shan Yu.

\section{REFERENCES}

Baidya DK, Khanna P, Maitra S. 2014. Analgesic efficacy and safety of thoracic paravertebral and epidural analgesia for thoracic surgery: a systematic review and meta-analysis. Interact Cardiovasc Thorac Surg 18:626-35.

Bayman EO, Parekh KR, Keech J, Selte A, Brennan TJ. 2017. A prospective study of chronic pain after thoracic surgery. Anesthesiology 126:938-951.

Bellieni CV, Cordelli DM, Raffaelli M, Ricci B, Morgese G, Buonocore G. 2006. Analgesic effect of watching TV during venipuncture. Arch Dis Child 91:1015-7.

Bigeleisen PE, Goehner N. 2015. Novel approaches in pain management in cardiac surgery. Curr Opin Anaesthesiol 28:89-94.

Boric K, Jelicic Kadic A, Boric M. 2019. Outcome domains and pain 
outcome measures in randomized controlled trials of interventions for postoperative pain in children and adolescents. Eur J Pain 23:389-96.

Chow CH, Van Lieshout RJ, Schmidt LA, Dobson KG, Buckley N. 2016. Systematic review: audiovisual interventions for reducing preoperative anxiety in children undergoing elective surgery. J Pediatr Psychol 41:182-203.

Custódio NB, Costa FDS, Cademartori MG, da Costa VPP, Goettems ML. 2020. Effectiveness of virtual reality glasses as a distraction for children during dental care. Pediatr Dent 42:93-102.

Faul F, Erdfelder E, Lang AG, Buchner A. 2007. G*Power 3: a flexible statistical power analysis program for the social, behavioral, and biomedical sciences. Behav Res Methods 39:175-91.

Garra G, Singer AJ, Taira BR. 2010. Validation of the Wong-Baker FACES Pain Rating Scale in pediatric emergency department patients. Acad Emerg Med 17:50-4.

Hammond SI. 2014. Children's early helping in action: Piagetian developmental theory and early prosocial behavior. Front Psychol 5:759.

Hole J, Hirsch M, Ball E, Meads C. 2015. Music as an aid for postoperative recovery in adults: a systematic review and meta-analysis. Lancet 386:1659-71.

Houdé O, Pineau A, Leroux G, et al. 2011. Functional magnetic resonance imaging study of Piaget's conservation-of-number task in preschool and school-age children: a neo-Piagetian approach. J Exp Child Psychol 110:332-46.

Kortesluoma RL, Nikkonen M, Serlo W. 2008. You just have to make the pain go away"--children's experiences of pain management. Pain Manag Nurs 9:143-9, 149.e1-5.

Lin CL, Hwang SL, Jiang P, Hsiung NH. 2020. Effect of music therapy on pain after orthopedic surgery-a systematic review and meta-analysis. Pain Pract 20:422-36.

Malviya S, Voepel-Lewis T, Burke C, Merkel S, Tait AR. 2006. The revised FLACC observational pain tool: improved reliability and validity for pain assessment in children with cognitive impairment. Paediatr Anaesth 16:258-65.

Marshall K, McLaughlin K. 2020. Pain management in thoracic Surgery. Thorac Surg Clin 30:339-346

Oliveira NC, Santos JL, Linhares MB. 2017. Audiovisual distraction for pain relief in paediatric inpatients: A crossover study. Eur J Pain 21:178-87.
Ozdemir S, Parlakyıldız Gokce A, Unver T. 2020. Simulation of three intraoral radiographic techniques in pediatric dental patients: subjective comfort assessment using the VAS and Wong-Baker FACES Pain Raiting Scale. BMC Oral Health 20:33.

Salmon K, McGuigan F, Pereira JK. 2006. Brief report: Optimizing children's memory and management of an invasive medical procedure: the influence of procedural narration and distraction. J Pediatr Psychol 31:522-7.

Savva DA, Kishk OA, Morgan JA, Biggs JM, Seung H, Bauer C. 2019. Post-operative non-steroidal anti-inflammatory drug use for pain in infant and paediatric cardiac surgery patients. Cardiol Young 29:1440-4.

Scohy TV, Golab HD, Egal M, Takkenberg JJ, Bogers AJ. 2011. Intraoperative glycemic control without insulin infusion during pediatric cardiac surgery for congenital heart disease. Paediatr Anaesth 21:872-9.

Voepel-Lewis T, Merkel S, Tait AR, Trzcinka A, Malviya S. 2002. The reliability and validity of the Face, Legs, Activity, Cry, Consolability observational tool as a measure of pain in children with cognitive impairment. Anesth Analg 95:1224-9.

Von Baeyer CL, Spagrud LJ. 2007. Systematic review of observational (behavioral) measures of pain for children and adolescents aged 3 to 18 years. Pain. 127:140-50.

Weydert JA, Shapiro DE, Acra SA, Monheim CJ, Chambers AS, Ball TM. 2006. Evaluation of guided imagery as treatment for recurrent abdominal pain in children: a randomized controlled trial. BMC Pediatr $6: 29$.

Wojtyś ME, Wąsikowski J, Wójcik N. 2019. Assessment of postoperative pain management and comparison of effectiveness of pain relief treatment involving paravertebral block and thoracic epidural analgesia in patients undergoing posterolateral thoracotomy. J Cardiothorac Surg 14:78.

Yap R, Nassif G, Hwang G, et al. 2020. Achieving opioid-free major colorectal surgery: Is it possible? Dig Surg 30:1-7.

Ziehm S, Rosendahl J, Barth J, Strauss BM, Mehnert A, Koranyi S. 2017. Psychological interventions for acute pain after open heart surgery. Cochrane Database Syst Rev 12:CD009984.

Zieliński J, Morawska-Kochman M, Zatoński T. 2020. Pain assessment and management in children in the postoperative period: A review of the most commonly used postoperative pain assessment tools, new diagnostic methods and the latest guidelines for postoperative pain therapy in children. Adv Clin Exp Med 29:365-74. 